\title{
Big Data, Data Science and Emerging Analytic tools : Impact in social science
}

\author{
Satabdi Saha ${ }^{1 *}$ | Tapabrata Maiti ${ }^{1 *}$
}

${ }^{1}$ Department of Statistics and Probability, Michigan State University, East Lansing, Michigan, 48824, USA

\section{Correspondence}

Satabdi Saha, Department of Statistics and Probability, Michigan State University, Wells Hall, 619 Red Cedar Rd, Room c413, East Lansing, MI 48824, USA

Email: sahasata@msu.edu

Funding information
Rapid advancement of the Internet and Internet of Things have led to companies generating gigantic volumes of data in every field of business. Big data research has thus become one of the most prominent topic of discussion garnering simultaneous attention from academia and industry. This paper attempts to understand the significance of big data in current scientific research and outline its unique characteristics, otherwise unavailable from traditional data sources. We focus on how big data has altered the scope and dimension of data science thus making it severely interdisciplinary. We further discuss the significance and opportunities of big data in the domain of social science research with a scrutiny of the challenges previously faced while using smaller datasets. Given the extensive utilization of big data analytics in all forms of socio-technical research, we argue the need to critically interrogate its assumptions and biases; thereby advocating the need for creating a just and ethical big data world.

\section{KEYWORDS}

Big Data, Data Science, Social science, Artificial Intelligence,

Twitter, social media, Healthcare 


\section{1 | INTRODUCTION}

Have you ever wondered what makes data science one of the most promising career paths? If you have, you are not alone. According to the McKinsey Global Institute [1], being a data scientist is currently the best job in America, with an average base salary of $\$ 105,395$ and there will be 4 to 5 million jobs requiring data analysis skills, and that large numbers of positions will only be filled through training or retraining. So what has led to the emergence of data science as one of the most sought-after disciplines? The answer lies in data becoming omnipresent. As increasing amounts of data become more accessible, there arises a growing need to understand, process, visualize and derive meaning from the data. Secondly decision-making has become data-driven, helping us seek out the subtleties of patterns hitherto hidden and using that information for organizational improvement and effective resource allocation. At the same time, availability of large quantities of data in the domain of social systems has given us an impetus to solve complex and dynamic problems related to social structures and human decisions.

\section{DATA AND DATA SCIENCE}

\section{1 | Traditional data}

Traditional data is data that is structured and can be stored in databases and analyzed using a personal computer; often containing numeric or text values represented in a tabular format. We use the term "traditional" to distinguish earlier forms of data from the current big data types. This type of data can be processed, analyzed and visualized using existing statistical tools or standard software. To gain a perspective about how a traditional dataset would look like and how we would generally analyze it, let us look at the Columbus crime data example [2]. This dataset contains information about the number of crimes (including residential burglaries and vehicle thefts) and possible determinants of crime recorded in Columbus, Ohio. In this example we want to investigate the dependence of crime rate on household income and house value. Here we work with a small part of the data containing information on 9 neighbourhoods (the complete dataset contains information on 49 neighborhoods).

\begin{tabular}{||cccccc||}
\hline Neighborhood & crime & household income & house value & x-coordinate & $y$-coordinate \\
\hline \hline 1 & 18.802 & 21.232 & 44.657 & 35.62 & 42.38 \\
\hline 2 & 32.388 & 4.477 & 33.2 & 36.5 & 40.52 \\
\hline 3 & 38.426 & 11.337 & 37.125 & 36.71 & 38.71 \\
\hline 5 & 0.178 & 8.438 & 75 & 33.36 & 38.41 \\
\hline 6 & 15.726 & 19.531 & 80.467 & 38.8 & 44.07 \\
\hline 7 & 30.627 & 15.956 & 26.35 & 39.82 & 41.18 \\
\hline 8 & 50.732 & 11.252 & 23.225 & 40.01 & 38 \\
\hline 9 & 26.067 & 16.029 & 28.75 & 43.75 & 39.28 \\
\hline 48.585 & 9.873 & 18 & 39.61 & 34.91 \\
\hline
\end{tabular}

The simplest solution will be to run a regression equation with crime rate as the response depending on predictors house value and household income.

$$
Y_{n}=X_{n} \beta+\epsilon_{n}
$$


where $Y_{n}$ represents the response vector of crime rates, $X_{n}$ is the design matrix of predictors house income and house value, $\beta$ is the vector of parameters and $\epsilon_{n}$ is the error vector. For this model we assume an independent error structure and the errors are also assumed to have a distribution independent of the covariates. On running this model in $\mathrm{R}$, we obtain parameter estimates for house value and household income as $\hat{\beta}_{\text {HOV AL }}=-0.2739$ and $\hat{\beta}_{I N C}=-1.5973$ respectively. The results indicate that neighborhoods with high income families and high priced homes have lower crime rates. It is important to note that we haven't used all the available variable information for our model such as the location coordinates. Thus to refine our analysis a little further we define a more structured regression model with a spatially correlated error structure. The regression model with spatial autoregressive (SAR) [3] disturbance units $u_{n}$ is specified as

$$
\begin{aligned}
& u_{n}=\rho M_{n} u_{n}+\epsilon_{n} \\
& Y_{n}=X_{n} \beta+u_{n}
\end{aligned}
$$

where $M_{n}$ is the known spatial weight matrix, and $M_{n} Y_{n}$ is the "spatial lag". This regression model accounts for the possible spatial correlations in neighborhoods instead of assuming that every neighborhood exists independently of the others. This results in changed parameter estimates, $\hat{\beta}_{H O V A L}=-1.17$ and $\hat{\beta}_{I N C}=-0.30$, now indicating that house value is a stronger indicator of crime rate as compared to higher income in a particular neighborhood. This result is in contrast with the result obtained while ignoring the spatial weights. Thus clearly the distance between neighborhoods plays an important role and should be valued while modelling the data. This example clearly proves that availability of more relevant domain information can guide a statistician to choose better models thus leading to precise analysis and inference. However the question remains whether more information implies better information? Not necessarily. It is important to note in this example as well as for all data analysis projects that the main idea lies in understanding the research question at hand and then to objectively determine what variables in the present data need to be analysed to best answer the question, rather than blindly incorporating information into a model. In this model, for example, it turns out that incorporating selective spatial information leads to better analysis and inference.

\section{2 | Challenges of Traditional data}

Now that we have discussed an example and seen how having access to more relevant information can lead us to better results, it now becomes imperative to investigate why the world seems to be moving away from traditionally small datasets and simpler statistical models. Traditionally most experiments involving primary research, for example in the fields of medicine, psychology etc. small samples were the norm. Experiments involving real people answering questionnaires or participating in clinical trials are often very expensive and time consuming leading to collection of smaller sized datasets. Also before the growth in the Internet of Things (IoT), which allows data to stream in to businesses at an unprecedented speed, tech data collection and storage was hard and expensive, thus constraining scientists to work with smaller samples and lesser variables. The issues that arises from working with smaller datasets are:

- Sample size - Having a smaller sample size can lead to problems of overfitting in the model. There are also problems of not having enough test and training sets for parameter tuning and increased proportion of measurement errors and missing values leading to faulty analysis. It also contains sampling bias if the data is not collected from a carefully controlled design. In this scenario, it becomes crucial to carefully clean the data, rely heavily on simpler models to reduce the amount of parameters to be estimated and to resort to the use of ensemble models using bootstrapping 
approaches for obtaining better results.

- Variable information - Having data on more variables can sometimes enrich a model, if the variables are carefully chosen. For example, information available from abundant health data amassed from numerous sources including electronic health records (EHRs), medical imaging, genomic sequencing, wearables, and medical devices has hugely enriched healthcare analytics by expanding diagnostic services, reducing prescription errors and identifying patient cohorts with greater risks of illnesses. These discoveries were limited in the era when models had to be designed using traditional electronic medical and human health data [4].

\section{NEW ERA OF BIG DATA AND DATA SCIENCE}

\subsection{What is Big Data?}

Big data is a term applied to data sets whose size or type is beyond the ability of traditional relational databases to capture, manage and process the data with low latency. Big data has one or more of the following characteristics: high volume, high velocity or high variety [5]. Artificial intelligence (AI), mobile, social and the loT are driving data complexity through new forms and sources of data. For example, big data comes from sensors, devices, video/audio, networks, log files, transactional applications, web, and social media - much of it generated in real time and at a very large scale.

\subsection{What is Data Science?}

Data science, also known as data-driven science, is an interdisciplinary field about scientific methods, processes and systems to extract knowledge or insights from data in various forms, either structured or unstructured, similar to data mining [6]. Figure 1 represents the five stages of the data science life cycle. With the advent of big data, the need to meaningfully organize and process diverse and unstructured datasets from a multitude of sources has grown substantially and so has the need for skilled data scientists. This has led to a growing number of academic programs in data science. There are currently more than 500 programs around the world, with most of them being in the United States. These programs mostly go by the names of data science, business analytics and prediction analytics and emphasizes on building a strong quantitative background in statistics, linear algebra as well as programming knowledge with focuses in data warehousing, mining, and modeling to build and analyze algorithms. Successful data scientists are able to pinpoint relevant research questions, collect, process and analyze data from a myriad of sources, provide novel data driven solutions and effectively communicate these findings so as to shape positive business decisions. Data science has created an interdisciplinary haven bringing together cutting edge research from a multitude of scientific disciplines, and binding them to successfully resolve the most challenging and pertinent questions of the 21 'st century, thus enriching every field of science, commerce, industry and government in the process.

\subsection{How is big data shaping data science?}

According to Data never sleeps $5.0[8]$

"Data is on overdrive. It's being generated at break-neck pace, flooding out of the dozens of connected devices we use every day, and it shows no signs of slowing down. In fact, the number of internet users has grown over a billion in the last five years, more than half of the world's web traffic now comes from mobile phones." 


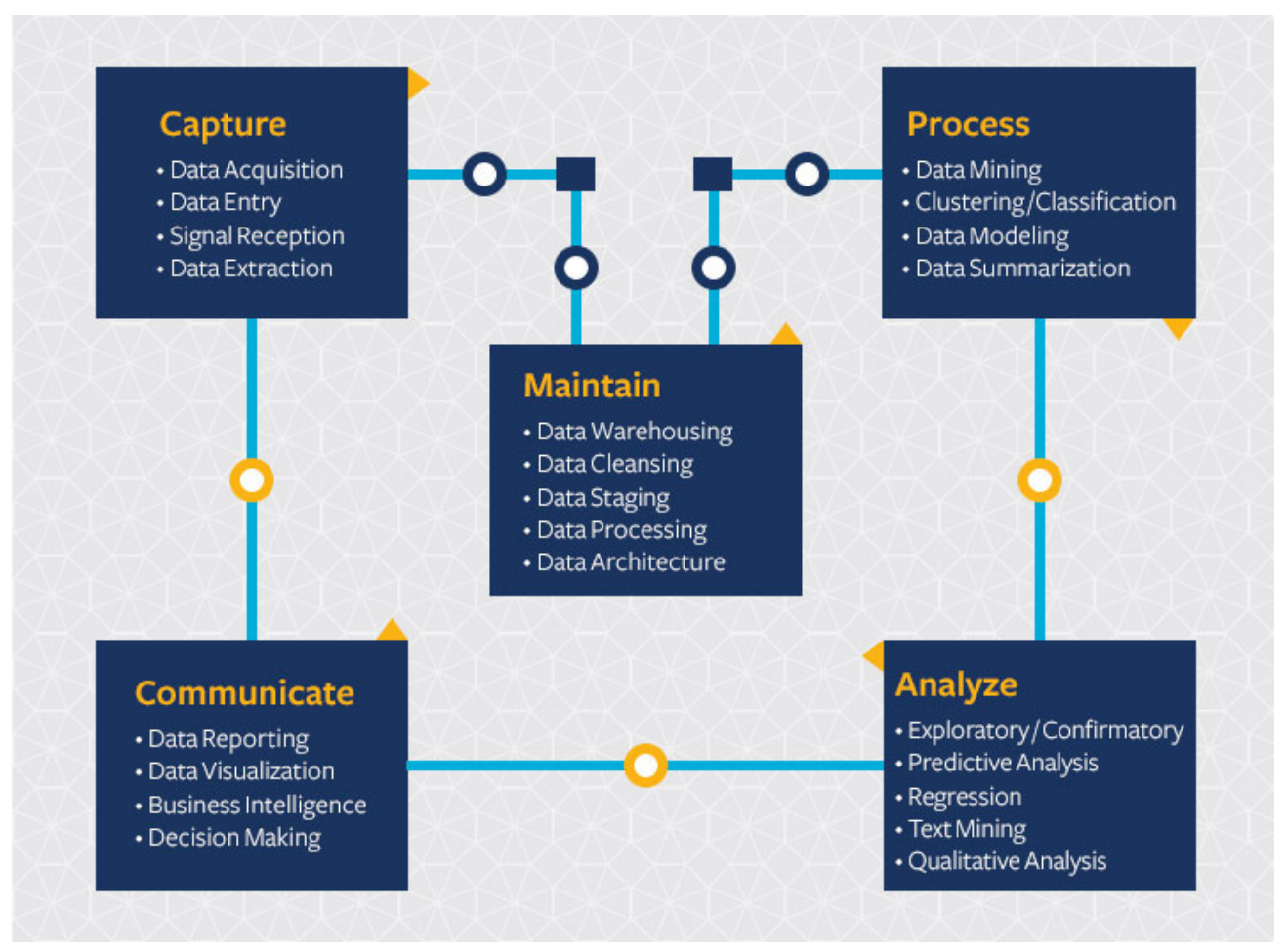

FIGURE 1 The image represents the five stages of the data science life cycle [7]. 
In the wake of such a data overdrive, there has arisen an acute need to process, understand and effectively interpret this massive data to solve critical problems in business hence fuelling enterprise growth and innovation. This has pushed demand for novel unconventional solutions that accelerate Artificial Intelligence (AI) and data science. To quote New Vantage Partners Big Data Executive Survey 2018 [9];

"Big Data and Al projects have become virtually indistinguishable, particularly given that machine learning is one of the most popular techniques for dealing with large volumes of fast-moving data. It's also the case that statistical approaches to AI-deep learning, for example-are increasingly popular. Therefore, we view traditional data analytics, Big Data, and Al as being on a continuum."

Thus big data has pushed data science outside the realm of standard statistical modelling. Increasingly complex black box models like neural nets and deep learning structures are becoming the norm in the domain of big data analytics. This new concept of data science requires data preparation, feature engineering, machine learning, and the production of a complete data science pipeline.

\section{4 | BIG DATA AND DATA SCIENCE IN SOCIAL SCIENCE RESEARCH}

Statistics has always played an integral role in social sciences with a heavy presence in fields of psychology, political science, sociology and anthropology. From analysing economic indicators, designing psychometric tests or investigating election trends, statistical theory has been extensively used in the domain of social research. Recently an increase in the capacity to collect and analyze massive amounts of data pertaining to these fields has created a need for a data driven computational social science construct that binds together domain knowledge, computational skills and extensive statistical knowledge. In this regard we discuss some of the emerging questions in social research and their potential data driven solutions. This is by no means an exhaustive list of the applications of big data and data science in social sciences, but rather just a glimpse into the world of endless possibilities.

\subsection{Some emerging foci in social research}

- Healthcare: Using MRI imaging data to develop effective models for early detection of Alzheimer's disease (AD) in patients, slowing down AD progression once it occurs, and improving quality of life and care for patients and their families.

- Political Research: Tracing the sentiments of tweets and hashtags to predict the political opinions of an electorate.

- Psychology: Address questions about the nature of visual search that pose intractable problems for laboratorybased research.

\subsection{Analytic solutions using big data}

- Healthcare: As of 2013, an estimated 44.4 million people had dementia worldwide, and this number is projected to reach 75.6 million in 2030 and 135.5 million in 2050 with most of the increase occurring in developing countries [10] Alzheimer's disease (AD) is the most common type of dementia constituting about $60-80 \%$ of the cases. To control this exponential growth in the number of $A D$ cases there is a pressing need to develop novel methodologies for early detection of $A D$ and slowing down its progression, once it's detected. Traditionally it has been difficult to 
conduct research in $A D$ due to the difficulties in recruitment and retention of patients in clinical research and the durations and costs associated with clinical trials. As a possible cure, researchers have resorted to providing data driven solutions. In this regard, big data has been identified as an invaluable resource by the AD research community for usage in the development of innovative technogies aiding AD research. There has been several primary foci of research including diagnosing $A D$ or mild cognitive impairment $(\mathrm{MCl})$, predicting $\mathrm{MCl}$ to $A D$ conversion, stratifying risks for $A D$, mining the literature for knowledge discovery, predicting $A D$ progression, describing clinical care for persons with $A D$, and understanding the relationship between cognition and $A D$ [11] [12]. The datasets used for $A D$ research include Alzheimer's Disease Neuroimaging Initiative (ADNI), electronic health records (EHR), MEDLINE, and other research datasets. Various data analytic methods ranging from data mining, machine learning, natural language processing (NLP), text mining to statistical and topological analysis have been used for researching $A D$ using these massive data sources leading to several novel results. To quote one, researchers from Stanford University [13] have recently developed a deep learning framework for diagnosis of AD. A 3D convolutional neural network CNN) was trained on MR images obtained from ADNI. This 3D CNN binary classification model was able to classify $A D$ and normal controls(NC) subjects with $94.1 \%$ accuracy. They further fine-tuned the model to develop a learning transfer strategy for classification of $\mathrm{MCl}$ subjects. Doing so, they built a three-class classifier to classify NC's, MCl's and AD. This three class classification model had an accuracy of $61.1 \%$ which was a large improvement over the accuracy delivered by current state of the methods and in line with accuracy of manual examination of MRI images. Their results also indicated that the hippocampus region of the brain is critical in the diagnosis of AD.

- Political Research: Election forecasting has long been a subject of interest to political scientists. The highly quantitative nature of the process provides ample opportunities for exploration and inference. Traditionally presidential election forecasting depended on data collected from previous presidential elections on predictor variables such as the candidate's approval rating, party affilation, campaign spending, commerce department's estimate of the annual growth rate of real gross domestic product (GDP) etc [14]. Well founded statistical models using time aggregated data on such social, political and economic variables formed the foundation of election forecasting research. These models often did not directly measure the political preferences of the voters. To delve directly into the political psyche of the voters, more and more political scientists are now relying on data gathered from social networking sites, which have become the new hub of political communication. Social networking sites generate massive amounts of digital data on human interactions every day. Twitter alone is a massive data stream, generating 200 billion tweets per year. Analysing Twitter data to predict election results can yield promising results for several reasons. When compared to traditional surveys, social media allows cheap and fast access to data which can be used to monitor an electoral campaign on a daily basis. Consequently it becomes easier to measure trends in real time and allow faster capture of changes in public opinion when compared to traditional polls. Therefore a growing number of political scientists are relying on Twitter data to measure political preferences. One of the machine learning techniques used to analyze public opinion is sentiment analysis. It is the process of decoding the emotion underlying a bunch of words. It helps to decipher the attitudes, opinions and emotions expressed within an tweet. It has been shown to provide reliable results in election forecasting in several studies. To quote one such study [15] uses a upervised and aggregated sentiment analysis method to monitor election campaigns. In this method, first the tweets are categorized into positive and negative opinions according to the sentiments they convey. This information is then fed into the algorithm as the training set to help it learn to define positive and negative text so it can be used to classify unread documents. The authors use this method to predict the outcome of the 2012 US presidential elections. They analyzed more than 50 million tweets expressing voting intentions for the four presidential candidates for a period of 40 days from September 28 to November 6, 2012. The analysis clearly 
indicated a positive sentiment towards Obama and further predicted a final victory for Obama with a clear and safe margin of 3.5\%. This proved to be highly accurate as the real gap in the share of votes was $3.9 \%$ and this was a great improvement over traditional survey polls which predicted that Obama would win by a margin of only $0.7 \%$.

- Psychology: Visual search is the task of searching for specific target items in an environment cluttered with distractors. Though the process may seem fairly common to the human eye, recreating visual search abilities in machines is a highly challenging task and decades of research has thus been dedicated to successfully decipher the mechanism behind it. Customary visual search experiments conducted in a lab environment requires multiple human subjects to perform several trials of visual search tasks for hundreds of experimental sessions. The obvious tediousness and expense associated with such a data generating process has pushed researchers to crowd source experiments to obtain requisite amounts of cheap data in short amounts of time. Having easy access to such big data can help cognitive scientists to answer several nuanced questions in the visual search domain with greater statistical power. To quote one such study, researchers from Duke University [16] partnered with Kedlin Co., the creators of Airport Scanner (https://www . airportscannergame.com), to collect data for researching identification of rare and ultra rare items from a cluster. In Airport Scanner, players are tasked with searching for illegal items in simulated $x$-ray bag images in an airport security environment while being provided with a logbook of illegal and legal items, which expands as they progress through the game. Armed with a massive dataset generated from nearly 2 billion trials of the online game, the researchers explored how appearance of rare target affects visual search performance. There were a total of 30 targets, defined as "ultra-rare" targets, in the data which appeared is less than $0.15 \%$ of the trials. Due to data availabilty contraints, it would have been practically impossible to investigate even the effect of one such rare target on visual search performance in a laboratory setting. However, with the large Airport Scanner dataset, the researchers were able to look at hundreds of cases for each of the nearly 30 "ultra-rare" targets. Thus a question that could have taken decades to answer in a laboratory setting became explorable using big data in a fraction of the time.

\section{5 | LIMITATIONS OF BIG DATA}

From all the examples that we have discussed so far we can safely postulate that currently big data is the fuel powering innovation in all domains of scientific research. Currently big data analytics is increasingly becoming the basis on which significant theories are developed and scientific decisions are made. However potential benefits always come riddled with challenges and risks. In this section we examine the issues associated with using big data analytics in scientific research with special attention to its validity, security and ethics.

To realize whether big data is really making a difference, it becomes mandatory to validate the results generated from big data analytic models, and to understand how much of a role big data has in generation of such results. We need to ask whether we are translating this massive information into real knowledge or using smaller datasets would provide similar results. It is also important to recognize that if similar or better research insights can be found using small data, then spending resources for collection, storage, processing and inference of such massive information actually serves no purpose. In some cases, focusing just on a single individual can be extraordinarily valuable. [17] remarks that "Big Data provides 'destablising amounts of knowledge and information that lack the regulating force of philosophy". Therefore ideally one should endeavour to integrate experimentally generated scientific evidence with the predictive power of big data to enable a shift from information to wisdom. One proposal for such integration in the domain of AD research has been advanced by [18], who suggested that data-driven analytic approaches "need to be organically integrated into a quantitative understanding of the pathology". Any new research which assumes that big data can generate 
knowledge independently without the requirement of discipline based expertise [19] should be critically examined for appropriateness. Also it is important to remember that big data does not refer to complete data. When one is for example analysing data from Twitter, they should keep in mind that not all socio-economic backgrounds are represented in Twitter and neither does every user involve in active participation. There are people with varying account types in Twitter from active twitterati to passive listeners. Also several accounts are 'bots' that produce automated content, to provoke specific emotions from tweeps. Therefore problems of context specificness, veracity and representation become rampant.

The other particular concern about big data usage is data privacy. The immense pervasiveness of big data has raised serious concerns about invasions of privacy and placed stark limitations on personal freedom, one related example being data stored in social network servers. How is private information gathered from million individuals getting used and is it invading our privacy? Internet giants like Google, Amazon, Facebook want to deliver highly personalized services and solutions and big data makes that possible. However, the massive power of big data technology is easing consumer identification and allowing wrongful user manipulation. One example that immediately comes to mind is the Facebook-Cambridge Analytica data scandal in early 2018, in which it was revealed that Cambridge Analytica had gathered the personal data of millions of peoples' Facebook profiles without their consent and used it for political advertising purposes. "While Cambridge Analytica claimed it had only collected 30 million Facebook user profiles,Facebook later confirmed that it actually had data on up to 87 million users, with 70.6 million of those people from the United States. Within the United States, Facebook estimated that California was the most affected U.S. state, with 6.7 million impacted users, followed by Texas, with 5.6 million, and Florida, with 4.3 million." [20]

Ethical usage of big data has always been a topic of debate in the research community. As White House reports [21] have indicated, big data can create the risk of unintentional discrimination. Big data models are being used extensively to guide decision making in several importance spheres like insurance companies, employers, college admissions officials etc. Since their databases contain information about customer demographics brands may unwittingly develop algorithms that penalize people based on ethnicity, gender or age.

“The Obama Administration's Big Data Working Group released reports on May 1, 2014 and February 5, 2015. These reports surveyed the use of data in the public and private sectors and analyzed opportunities for technological innovation as well as privacy challenges. One important social justice concern the 2014 report highlighted was "the potential of encoding discrimination in automated decisions"-that is, that discrimination may "be the inadvertent outcome of the way big data technologies are structured and used." [22]

In this context [23] raise several critical concerns.
"Very little is understood about the ethical implications underpinning the Big Data phenomenon. Should someone be included as a part of a large aggregate of data? What if someone's 'public' blog post is taken out of context and analyzed in a way that the author never imagined? What does it mean for someone to be spotlighted or to be analyzed without knowing it? Who is responsible for making certain that individuals and communities are not hurt by the research process? What does informed consent look like?"

Therefore to promote ethical usage of big data technology, it is important that "researchers reflect on the importance of accountability: both to the field of research and to the research subjects" [23]. 


\section{6 | CONCLUSION: IS THE WORLD READY FOR A CHANGE?}

Big data has created a world of opportunities in the field of research, by advancing technology in terms of data storage, querying, processing and analytical inference. Tantalizing amounts of data combined with state of the art analytics have been able to generate significant results in important spheres ranging from robotics to healthcare. In the realm of social science research, big data holds great promise. Social scientists are utilizing big data for developing innovative methods to answer challenging research questions. They are collaborating with computer scientists and statisticians to succesfully merge artificial intelligence (Al) and social science perspectives with the intention of identifying latent patterns in causal inference. Big data has also shown enough potential in enhancing and strengthening existing theory, methods and inference. Thus merging the computational prowess of big data analytics with the insights of a social scientist can bring about revolutionary developments in this field. However these promises of innovation come tied with several difficulties. It has become more important than ever to question the assumptions, benefits and risks associated with this new wave of research. It is important to verify the sources of big data, to judge whether it provides an inclusive representation without bias towards a certain group of people and whether it measures the contextually correct objective in addition to giving better solutions. Delving into such questions will facilitate a deeper understanding of big data analytics and create an environment of pertinent and ethical research which will finally lead to promising ways of converting information to actionable knowledge.

\section{REFERENCES}

[1] Manyika J. Big data: The next frontier for innovation, competition, and productivity. http://www mckinsey com/Insights/MGI/Research/Technology_and_Innovation/Big_data_The_next_frontier_for_innovation 2011;

[2] Anselin L. Spatial Econometrics: Methods and Models, vol. 4. Springer Science \& Business Media; 1988.

[3] Whitle P. On stationary process in the plane. Biometrika 1954;41(3-4):434-449.

[4] Catalyst N. Healthcare big data and the promise of value-based care. NEJM Catalyst 2018;.

[5] Doug Laney, Application Delivery Strategies; 2001. https://blogs.gartner.com/doug-laney/files/2012/01/ad9493D-Data-Management-Controlling-Data-Volume-Velocity-and-Variety.pdf.

[6] Wikipedia contributors, Data science - Wikipedia, The Free Encyclopedia; 2019. [Online; accessed 23-December-2019]. https://en.wikipedia.org/w/index.php?title=Data_science\&oldid=931025700.

[7] datascience@berkely, What is Data Science?; 2018. [Online; accessed 23-December-2019]. https://en.wikipedia. org/w/index . php?title=Data_science\&oldid=931025700.

[8] DOMO, Data Never Sleeps 5.0; 2017. [Online; accessed 23-December-2019]. https://www.domo.com/learn/datanever-sleeps-5.

[9] LLC NP. Data and Innovation: How Big Data and Al are Driving Business Innovation 2018;https : //newvantage . com/wpcontent/uploads/2018/01/Big-Data-Executive-Survey-2018-Findings-1.pdf.

[10] Alzheimer's Disease International, Dementia statistics; 2015. [Online; accessed 23-December-2019]. https ://www . alz. co.uk/research/statistics.

[11] Zhang R, Simon G, Yu F. Advancing Alzheimer's research: A review of big data promises. International journal of medical informatics 2017;106:48-56. 
[12] Li Y, Zhang L, Bozoki A, Zhu DC, Choi J, Maiti T, et al. Early prediction of Alzheimer's disease using longitudinal volumetric MRI data from ADNI. Health Services and Outcomes Research Methodology 2019 Dec;https: //doi . org/10.1007/ s10742-019-00206-3.

[13] Esmaeilzadeh S, Belivanis DI, Pohl KM, Adeli E. End-to-end Alzheimer's disease diagnosis and biomarker identification. In: International Workshop on Machine Learning in Medical Imaging Springer; 2018. p. 337-345.

[14] Abramowitz Al. Bill and Al's excellent adventure: Forecasting the 1996 presidential election. American Politics Quarterly 1996;24(4):434-442.

[15] Ceron A, Curini L, lacus SM. Using sentiment analysis to monitor electoral campaigns: Method matters-evidence from the United States and Italy. Social Science Computer Review 2015;33(1):3-20.

[16] Mitroff SR, Biggs AT, Adamo SH, Dowd EW, Winkle J, Clark K. What can 1 billion trials tell us about visual search? Journal of experimental psychology: human perception and performance 2015;41(1):1.

[17] Berry D. The computational turn: Thinking about the digital humanities. Culture machine 2011;12.

[18] Geerts H, Dacks PA, Devanarayan V, Haas M, Khachaturian ZS, Gordon MF, et al. Big data to smart data in Alzheimer's disease: The brain health modeling initiative to foster actionable knowledge. Alzheimer's \& Dementia 2016;12(9):10141021.

[19] Anderson C. The end of theory: The data deluge makes the scientific method obsolete. Wired magazine 2008;16(7):1607.

[20] Wikipedia contributors, Facebook-Cambridge Analytica data scandal - Wikipedia, The Free Encyclopedia; 2019. [Online; accessed 24-December-2019]. https://en.wikipedia.org/w/index.php?title=Facebook\% $2 \% 80 \% 93$ Cambridge_ Analytica_data_scandal\&oldid=931529726.

[21] Executive Office of the President, Big Data: A Report on Algorithmic Systems, Opportunity,and Civil Rights; 2016. https : //www.whitehouse.gov/sites/default/files/microsites/ostp/2016_0504_data_discrimination.pdf.

[22] Kh R. Big Data Privacy Issues that Should Worry Every Internet User 2017; https : / www . smartdatacollective . com/bigdata-privacy-issues-worry-every-internet-user/.

[23] Boyd D, Crawford K. Critical questions for big data: Provocations for a cultural, technological, and scholarly phenomenon. Information, communication \& society 2012;15(5):662-679. 